\title{
Review of: "The prevalence and correlates of vision impairment and glasses ownership among ethnic minority and Han schoolchildren in rural China"
}

\author{
Alison Bruce ${ }^{1}$ \\ 1 Bradford Institute for Health Research
}

Potential competing interests: The author(s) declared that no potential competing interests exist.

The aim of this cross sectional study is to report the prevalence of visual impairment, defined as visual acuity (VA) $\leq 6 / 12$ in both eyes without glasses, in children aged 12-15 years attending Junior High School in one rural district, Ningxia, China. The authors are particularly interested in comparing differences in prevalence and reporting factors associated with visual impairment including ethnicity between the Han (majority population) and the Hui. Prevalence of visual impairment among the population was found to be high with a low rate of glasses ownership. Visual impairment was found to be slightly higher among the Han population and low rates of glasses ownership were reported for both groups, especially the Hui. The results of this study must be considered alongside its limitations.

- The study methodology utilizes a survey to collect both the child and parental data required to evaluate potential associated factors such as parental education, parental wealth, and time spent studying or watching TV. The questionnaire was completed by the child with the help of an enumerator. There is no description of the training given to the enumerators and no description of how the survey was administered. The questionnaire is not provided as an appendix and the reader cannot evaluate the potential for bias https://academic.oup.com/esr/article/31/3/312/437455 . The questions regarding the amount of time spent studying after school are problematic; firstly a child may want to please the enumerator by reporting an increased amount of study and a decreased amount of time watching TV after school, secondly these activities are almost interchangeable i.e. If the child is not watching TV they are studying, these factors combined in the analysis could potentially lead to collinearity. The measure of wealth used is "within the lowest tercile", it is questionable if a child would know the details of their parents income and may exaggerate household possessions. An appendix should be made available providing details of the methodology of the survey, the personnel and their training, the list of questions and the number of children who both completed both the VA test and the survey detailing any missing data.

- The authors state that only variables that were statistically significant $(p<0.05)$ in the univariate analysis were included in the multivariate regression model. However there are a number of variables where this significance level was not met yet the variables were included in the final model e.g. In the simple regression model (Table 2, Panel A), parental education was found to have no significance for 
both the Han population $(R R, 1.04 ; P=0.092)$ and the Hui population $(R R, 1.02 ; P=0.409)$ yet this variable was included in the final multi-variable analysis. Greater clarity regarding the analysis is required.

- The authors report visual impairment (VA without glasses) however refractive error prevalence is not reported. There is an assumption that the visual impairment (VI) is a result of uncorrected myopia, however populations with different ethnic origins may also demonstrate differing refractive characteristics, with the Yi population in Southwest China reported to have a higher risk of hyperopia than the Han population, perhaps related to time spent in rural areas https://bmcophthalmol.biomedcentral.com/articles/10.1186/s12886-019-1042-0. Therefore one cannot assume in this study that uncorrected myopia was the cause of the VI across both groups; refractive data is required to confirm this association.

Reporting the prevalence of visual impairment within a population and the associated factors, is important for understanding and managing a significant public health issue which has been shown to impact on educational attainment. This study's design and limitations restrict the generalizability of the findings. 